\title{
PROJECTIVE BITOPOLOGICAL SPACES II.
}

\author{
M. C. DATTA
}

(Received 12 October 1970; revised 16 March 1971)

Communicated by B. Mond

\section{Introduction}

Gleason [3] proved that in the category $\mathscr{G}$ of compact Hausdorff spaces and continuous maps, the projective objects are precisely the extremally disconnected spaces contained in the category. Strauss [7] generalised this and proved that in the category $\mathscr{S}$ of regular Hausdorff spaces and perfect maps the projective objects are again precisely the extremally disconnected ones. Observe that Gleason's category is a full subcategory of Strauss's category.

In our earlier paper [1] we generalised the above result of Gleason to the situation of bitopological spaces. The purpose of the present paper is to do the same for the above result of Strauss.

In this process we obtain some pleasant surprises. Let us make the convention that every topological space $(X, \tau)$ is also considered as a bitopological space $(X, \tau, \tau)$. Then the category of bitopological spaces that we obtained in [1], to be called cat $A$ in this paper, contains $\mathscr{G}$ as a full subcategory. We define another category of bitopological spaces in this paper, to be called cat $B$, and we determine the projectives in this category (cf. Main Theorem). Strauss's theorem comes out as a special case of our Main Theorem. Further it turns out that (1) $\mathscr{G}$ and $\mathscr{S}$ are both full subcategories of cat $B$; (2) cat $A$ is not contained in cat $B$; (3) the intersection of cat $A$ and cat $B$ is precisely $\mathscr{G}$; and (4) in $\mathscr{G}$, the two concepts "semicompact, quasi-Hausdorff, extremally disconnected bitopological spaces" and "pairwise regular, pairwise Hausdorff, pairwise extremally disconnected bitopological spaces"- the projective objects in cat $A$ and cat $B$ respectivelycoincide with the conventional extremally disconnected topological spaces. As a side result we prove a Tychonoff Theorem (cf. 4.9) for 'pairwise compactness'defined by Fletcher, Hoyle and Patty [2]-for bitopological spaces.

\section{Definitions and statement of main theorem}

Throughout let $(X, P, Q),\left(X_{1}, P_{1}, Q_{1}\right)$ and $\left(X_{2}, P_{2}, Q_{2}\right)$ stand for bitopological spaces. Any topological concept associated with the upper bound topology of 
$P$ and $Q$ is referred to as a property of $(X, P, Q)$ with the prefix 'semi'. Thus we get the concepts of semi-compactness, semi-open set, semi-closed set etc. A set $A \subset X$ is said to be quasi-open if for every $x \in A$ there exists either a $P$-open neighbourhood $U_{x} \subset A$ or a $Q$-open neighbourhood $V_{x} \subset A$. The complement of a quasi-open set is defined to be quasi-closed. The quasi-closure of any set $A$ turns out to be $A^{P} \cap A^{Q}$ where $A^{P}$ denotes the $P$-closure of $A$ and $A^{Q}$ is the $Q$-closure of $A$. $(X, P, Q)$ is said to be quasi-Hausdorff if given $x_{1} \neq x_{2}$, there exist disjoint quasiopen sets $U_{1}, U_{2}$ containing $x_{1}, x_{2}$ respectively. A map $X_{1} \rightarrow X_{2}$ is said to be quasi-continuous if the inverse image of every quasi-open set is quasi-open. $(X, P, Q)$ is said to be extremally disconnected if the quasi-closure of every semiopen set is quasi-open.

With these definitions we proved in [1] that in the category, cat $A$, of semicompact, quasi-Hausdorff bitopological spaces and quasi-continuous maps the projective spaces are precisely the extremally disconnected ones.

In order now to generalize Strauss's theorem we recall the following definition of Kelly [4] and Fletcher, Hoyle and Patty [2]. $(X, P, Q)$ is said to be pairwise Hausdorff if given $x_{1} \neq x_{2}$ there exists a $P$-open neighbourhood $U$ of $x_{1}$ and a $Q$-open neighbourhood $V$ of $x_{2}$ which are disjoint. $(X, P, Q)$ is said to be pairwise regular if given a $P$-closed ( $Q$-closed) set $A$ and $x \notin A$, there exist a $Q$-open ( $P$-open) set $U \supset A$ and a $P$-open ( $Q$-open) set $V$ containing $x$, which are disjoint. A family $\mathscr{A}$ of $P$-open and $Q$-open sets of $(X, P, Q)$ is said to be pairwise open if there exists at least one $P$-open set in $\mathscr{A}$ and at least one $Q$-open set in $\mathscr{A}$. If every pairwise open covering of $X$ has a finite subcovering, we say $(X, P, Q)$ is pairwise compact. A semi-compact space is always $P$-compact, $Q$-compact and pairwise compact. Now for the purpose of this paper we make the following two definitions.

(2.1) Definition. A mapping $f:\left(X_{1}, P_{1}, Q_{1}\right) \rightarrow\left(X_{2}, P_{2}, Q_{2}\right)$ is said to be perfect if

(i) $f$ is continuous, (cf. Pervin [6]); that is $f$ is $P_{1}-P_{2}$-continuous and also $Q_{1}-Q_{2}$-continuous,

(ii) $f$ is compact, that is, the inverse image of every point of $X_{2}$ is $P_{1}$-compact, $Q_{1}$-compact and pairwise compact; and

(iii) $f$ is closed; that is, the image of every $P_{1}$-closed $\left(Q_{1}\right.$-closed) subset of $X_{1}$ is $P_{2}$-closed $\left(Q_{2}\right.$-closed) subset of $X_{2}$.

(2.2) Definition. $(X, P, Q)$ is said to be pairwise extremally disconnected if the $Q$-closure of every $P$-open set is $P$-open and the $P$-closure of every $Q$-open set is $Q$-open.

Now we can state our

MAIN THEOREM. In the category, cat $B$, of pairwise regular, pairwise 
Hausdorff bitopological spaces and perfect maps, the projective spaces are precisely the pairwise extremally disconnected ones.

Throughout the rest of the paper " $p$ " will be used for "pairwise"; i.e. "p-regular" will mean "pairwise regular", and so on.

\section{Preliminaries}

(3.1) Lemma. Let $(X, P, Q)$ be a p-Hausdorff space. Let $U$ and $V$ be P-open and $Q$-open subsets of $X$ respectively such that $U \cap V=\varnothing$. Then $U^{Q} \cap V=\varnothing$ and $U \cap V^{P}=\varnothing$.

Proof. Suppose that $U^{Q} \cap V \neq \varnothing$. Let $x \in U^{Q} \cap V . x \in U^{Q}$ implies that every $Q$-open neighbourhood of $x$ meets $U$. But $V$ is a $Q$-open set containing $x$ which does not meet $U$. This contradiction shows that $U^{Q} \cap V=\varnothing$. Similarly, $U \cap V^{P}=\varnothing$.

We recall the following definition from [1]. Let $\left(X_{i}, P_{i}, Q_{i}\right)_{i \in I}$ be a family of bitopological spaces. On the product set $X=\pi_{i \in I} X_{i}$, we define a bitopological structure $(P, Q)$ by taking $P$ as the product topology generated by the $P_{i}$ 's and $Q$ as the product topology generated by the $Q_{i}$ 's.

(3.2) Proposition. Any product of p-Hausdorff spaces is p-Hausdor,j.

The proof is analogous to the usual proof of the proposition that the product of Hausdorff spaces is Hausdorff.

(3.3) Proposition. Any product of p-regular spaces is p-regular.

The proof runs along the same lines as in the case of topological spaces; i.e. the product of regular spaces is regular.

(3.4) Lemma. Let $(X, P, Q)$ be p-regular space. Let $A$ be P-compact (Q-compact) subset of $X$ and $B$ be P-closed (Q-closed) subset of $X$ such that $A \cap B=\varnothing$. Then $A$ and $B$ can be separated by P-open (Q-open) and Q-open $(P$-open) subsets of $X$ respectively.

Proof. Let $A$ be $P$-compact and $B$ be $P$-closed subset of $X$ such that $A \cap B=\varnothing$. By $p$-regularity of $X$, we have for each $a \in A$, a $P$-open set $U_{a}$ and a $Q$-open set $V_{a}$ such that

$$
a \in U_{a}, B \subset V_{a} \text { and } U_{a} \cap V_{a}=\varnothing
$$

$\left\{U_{a}\right\}_{a \in A}$ is a $P$-open covering of $A$ and since $A$ is $P$-compact there exists a finite subset $A_{1} \subset A$ such that

$$
A \subset \bigcup_{a \in A_{1}} U_{a}
$$


Let

$$
U=\bigcup_{a \in A_{1}} U_{a} \text { and } V=\bigcap_{a \in A_{1}} V_{a}
$$

Then $A \subset U, B \subset V$ and $U \cap V=\varnothing$. The other case is similar.

\section{4. p-compact spaces}

(4.1) Definition. A family $\mathscr{F}$ of a space $(X, P, Q)$ is said to be $p$-closed if some (at least one but not all) members of $\mathscr{F}$ are $P$-closed and the remaining members are $Q$-closed.

(4.2) Theorem. A space $(X, P, Q)$ is p-compact iff each family of p-closed subsets of $X$ with the finite intersection property, has a non-void intersection.

The proof follows in a standard way.

(4.3) Definition. A family $\mathscr{F}$ of subsets of a space $(X, P, Q)$ is called inadequate if the family does not cover $X$. The family is called finitely inadequate iff no finite subfamily of $\mathscr{F}$ covers $X$.

(4.4) Definition. A family $\mathscr{C}$ of subsets of a space $(X, P, Q)$ is called common if $\cap \mathscr{C} \neq \varnothing ; \mathscr{C}$ is finitely common iff each finite subfamily of $\mathscr{C}$ has a non-void intersection.

(4.5) Proposition. Let $(X, P, Q)$ be a bitopological space. Then the following are equivalent.

(a) each finitely inadequate family of p-open sets in $X$ is inadequate;

(b) each finitely common family of p-closed sets of $X$ is common.

The proof follows from definitions (4.3), (4.4) and De Morgan formulae.

REMARK. In view of theorem (4.2), conditions (a) and (b) are equivalent to $p$-compactness of $(X, P, Q)$.

The following lemmas 4.6, 4.7 and Theorems 4.8 and 4.9 are generalizations of known results in general topological spaces [5, p 79-80 and 126-128] and [8, p 127-129] to bitopological spaces.

(4.6) LEMMA. Let $\mathscr{F}$ be a finitely inadequate family of p-open subsets of $(X, P, Q)$. Then there is a maximal finitely inadequate family $\mathscr{D}$ of $p$-open subsets of $(X, P, Q)$ such that $\mathscr{F} \subset \mathscr{D}$.

Proof. Let $\mathscr{C}$ be the collection of all finitely inadequate families of $p$-open sets. Let $\mathscr{C}$ be ordered by set inclusion; i.e., $\mathscr{C}_{1}, \mathscr{C}_{2} \in \mathscr{C}$ then $\mathscr{C}_{1} \leqq \mathscr{C}_{2}$ if $\mathscr{C}_{1} \subset \mathscr{C}_{2}$. Now $\mathscr{F} \in \mathscr{C}$. So, by Hausdorff maximal principle, let $\mathscr{A}$ be a maximal linearly ordered subcollection of $\mathscr{C}$ such that $\mathscr{F} \in \mathscr{A}$. Let $\mathscr{D}=\cup \mathscr{A} . \mathscr{D}$ is a family of $p$-open 
sets, since each member of $\mathscr{A}$ is such a family. We shall show (i) $\mathscr{D}$ is finitely inadequate and (2) $\mathscr{D}$ is a maximal finitely inadequate family of $p$-open sets.

To prove (i), let $D_{1}, D_{2}, \cdots, D_{K} \in \mathscr{D}$. Then for each $i$, there is some $\mathscr{C}_{i}$ in $\mathscr{A}$ such that $D_{i} \in \mathscr{C}_{i}$ Since $\mathscr{A}$ is linearly ordered one of these $\mathscr{C}_{i}$, say $\mathscr{C}_{j}$, contains each of the other $\mathscr{C}_{i} s$. So $D_{i} \in \mathscr{C}_{j}$ for each $i$. Thus $\bigcup_{i=1}^{K} D_{i} \neq X$, since $\mathscr{C}_{j}$ is finitely inadequate. Hence $\mathscr{D}$ is finitely inadequate.

Now, suppose (2) were not true; i.e., suppose there were some open (either $P$-open or $Q$-open) set $G \notin \mathscr{D}$ such that $\mathscr{D} \cup\{G\}$ is still finitely inadequate. Then $\mathscr{A} \cup\{\mathscr{D} \cup\{G\}\}$ would be linearly ordered and would properly contain $\mathscr{A}$, contradicting the maximality of $\mathscr{A}$. Thus (2) must be true, and $\mathscr{D}$ is the required maximal finitely inadequate family containing $\mathscr{F}$.

(4.7) Lemma. Let $(X, P, Q)$ be a bitopological space. Let $\mathscr{D}$ be a maximal finitely inadequate family of p-open sets. If some member of $\mathscr{D}$ contains $G_{1} \cap G_{2} \cap \cdots \cap G_{n}$, where each $G_{i}$ is $P$-open (Q-open), then $G_{K} \in \mathscr{D}$ for some $K \in\{1,2, \cdots, n\}$.

Proof. First suppose $n=2$. Suppose $G_{1} \notin \mathscr{D}$ and $G_{2} \notin \mathscr{D}$. Then by maximality of $\mathscr{D}$, there must be members $A_{1}, A_{2}, \cdots, A_{m}$ of $\mathscr{D}$ such that

$$
G_{1} \cup A_{1} \cup A_{2} \cup \cdots \cup A_{m}=X .
$$

Also, there are members $B_{1}, B_{2}, \cdots, B_{n}$ of $\mathscr{D}$ such that

$$
G_{2} \cup B_{1} \cup B_{2} \cup \cdots \cup B_{n}=X .
$$

Then

$$
\left(G_{1} \cap G_{2}\right) \cup A_{1} \cup \cdots \cup A_{m} \cup B_{1} \cup \cdots \cup B_{n}=X,
$$

so that no members of $\mathscr{D}$ can contain $G_{1} \cap G_{2}$. Thus we have proved a contradiction for $n=2$. Similarly, we conclude the lemma for any positive integer $n$.

The following is the generalization of Alexander's theorem.

(4.8) THEOREM. Let $S_{P}$ be a subbase for $P$ and $S_{Q}$ be the subbase for $Q$, where $(X, P, Q)$ is a bitopological space. If each p-open cover of $X$ consisting of sets from $S_{P} \cup S_{Q}$ has a finite subcover, then $X$ is p-compact.

The proof follows from theorem (4.5), its remark and lemmas (4.6) and (4.7).

The following is the generalization of Tychonoff's theorem.

(4.9) THEOREM. The product of p-compact bitopological spaces is p-compact. Conversely, if the product is p-compact, then each component is p-compact.

Proof. Let $\left\{X_{\alpha}, P_{\alpha}, Q_{\alpha}\right\}_{\alpha \in \wedge}$ be a family of bitopological spaces. Let $(X, P, Q)$ be the product space. Suppose each $\left(X_{\alpha}, P_{\alpha}, Q_{\alpha}\right)$ is $p$-compact. Let $S_{P}$ be the defining subbase for $P$ and $S_{Q}$ be the subbase for $Q$. Then 


$$
S_{P}=\left\{\pi_{\alpha}^{-1}\left(U_{\alpha}\right): U_{\alpha} \in P_{\alpha}, \alpha \in \wedge\right\} ; \text { and } S_{Q}=\left\{\pi_{\alpha}^{-1}\left(V_{\alpha}\right): V_{\alpha} \in Q_{\alpha}, \alpha \in \wedge\right\} .
$$

Let $S=S_{P} \cup S_{Q}$ and let $\mathscr{F}$ be a $p$-open finitely inadequate subfamily of $S$. For $\alpha \in \wedge$, let

$$
\mathscr{B}_{\alpha}=\left\{W_{\alpha}: \pi_{\alpha}^{-1}\left(W_{\alpha}\right) \in \mathscr{F}\right\}
$$

Then $\mathscr{B}_{\alpha}$ is finitely inadequate in $X_{\alpha}$. Since $X_{\alpha}$ is $p$-compact, $\mathscr{B}_{\alpha}$ does not cover $X_{\alpha}$. So for each $\alpha \in \wedge$, let

$$
X_{\alpha} \in \cup\left\{B \mid B \in \mathscr{B}_{\alpha}\right\}^{\prime} .
$$

Let $x=\left(x_{\alpha}\right) \in X . \quad x \notin \cup \mathscr{F}$ (because $\left.x_{\alpha} \notin \cup\left\{B \mid B-\mathscr{B}_{\alpha}\right\}\right)$. Therefore $\mathscr{F}$ is inadequate. By theorem $(4.8),(X, P, Q)$ is $p$-compact.

The converse follows from the fact that projections are continuous and the continuous image of $p$-compact spaces is $p$-compact.

\section{Perfect mappings}

(5.1) We begin with an example of a perfect mapping.

Example. Let $X_{1}$ be the real line $R$, let $P_{1}$ be the usual topology and

$$
Q_{1}=\{\phi\} \cup\left\{U \cup(x, \infty): U \in P_{1} \text { and } x \in X_{1}\right\} .
$$

Let $X_{2}$ be also the real line $R$, let $P_{2}$ be the usual topology and let

$$
Q_{2}=\{\phi\} \cup\left\{U \cup(-\infty, x): U \in P_{2} \text { and } x \in X_{2}\right\} .
$$

Then $f:\left(X_{1}, P_{1}, Q_{1}\right) \rightarrow\left(X_{2}, P_{2}, Q_{2}\right)$ defined by $f(x)=-x$ for every $x \in X_{1}$, is a perfect mapping.

REMARK. Every continuous mapping between p-compact, p-Hausdorff spaces is perfect. For we have only to show (ii) and (iii). Let $x \in X_{2} \cdot\{x\}$ is both $P_{2}$-closed and $Q_{2}$-closed (because $X_{2}$ is $p$-Hausdorff) and so $f^{-1}(x)$ is both $P_{1}$-closed and $Q_{1}$-closed (because $f$ is continuous). Therefore $f^{-1}(x)$ is $P_{1}$-compact, $Q_{1}$ compact and $p$-compact. To show (iii), let $F$ be $P_{1}$-closed $\left(Q_{1}\right.$-closed) subset of $X_{1}$, then $F$ is $Q_{1}$-compact ( $P_{1}$-compact). Therefore $f(F)$ is $Q_{2}$-compact ( $P_{2}$-compact) and hence $P_{2}$-closed $\left(Q_{2}\right.$-closed) subset of $X_{2}$.

(5.2) LemMa. An image of a p-regular space under a perfect mapping is p-regular.

The proof runs on the same lines as that of lemma 3 of [7]. The following three lemmas are generalizations of Strauss's lemmas 4,5 and 6 respectively [7].

(5.3) LeMma. Let $f$ be a closed and compact mapping from a space $\left(X_{1}, P_{1}, Q_{1}\right)$ into a space $\left(X_{2}, P_{2}, Q_{2}\right)$. Then $f^{-1}$ maps all $P_{2}$-compact $\left(Q_{2}-\right.$ compact) sets to $P_{1}$-compact $\left(Q_{1}\right.$-compact $)$ sets and all p-compact sets of $X_{2}$ to p-compact sets of $X_{1}$. 
Proof. Let $C$ be any $p$-compact subset of $X_{2}$. We shall show that $f^{-1}(C)$ is $p$-compact subset of $X_{1}$. Suppose that there is a family $\left\{H_{\alpha}\right\}_{\alpha \in \wedge}$ of $p$-closed subsets of $X_{1}$ such that $\left\{H_{\alpha}\right\}_{\alpha \in \wedge}$ is filtered downwards,

$$
f^{-1}(C) \cap\left(\bigcap_{\alpha \in \lambda} H_{x}\right)=\varnothing
$$

but that for any $\alpha \in \wedge, f^{-1}(C) \cap H_{\alpha} \neq \varnothing$. Since $f\left(H_{\alpha}\right)$ is a $P_{2}$-closed or $Q_{2}$-closed set (depending on $H_{\alpha}$ ) which meets $C$, there exists a point $y \in C$ such that

$$
y \in \bigcap_{\alpha \in \Lambda} f\left(H_{\alpha}\right)
$$

(because $C$ is $p$-compact). For every $\alpha \in \wedge, f^{-1}(y) \cap H_{\alpha} \neq \varnothing$. Since $f^{-1}(y)$ is $p$-compact, there exists a point

$$
x \in f^{-1}(y) \cap\left(\bigcap_{\alpha \in \lambda} H_{\alpha}\right) .
$$

But $x \in f^{-1}(C)$ contradicting our assumption that

$$
f^{-1}(C) \cap\left(\bigcap_{\alpha \in \wedge} H_{\alpha}\right)=\varnothing .
$$

Similarly we can show that inverse image of every $P_{2}$-compact $\left(Q_{2}\right.$-compact) subset of $X_{2}$ is $P_{1}$-compact $\left(Q_{1}\right.$-compact) subset of $X_{1}$.

Remark. The above lemma shows that the composite of two perfect mappings is perfect. So, we may speak of the category cat $B$, of $p$-regular, $p$-Hausdorff spaces and perfect mappings.

(5.4) Lemma. Let $\left(X_{1}, P_{1}, Q_{1}\right)$ and $\left(X_{2}, P_{2}, Q_{2}\right)$ be bitopological spaces and let $f$ be a compact mapping from $X_{1}$ onto $X_{2}$. Then there is a quasi-closed subspace $F$ of $X_{1}$ such that $f(F)=X_{2}$ and such that no proper $P_{1}$-closed or $Q_{1}$-closed subspace of $F$ has this property.

Proof. Let $\Gamma$ be any linearly ordered $p$-closed family of subsets of $X_{1}$ which are mapped onto $X_{2}$ by $f$. Then $F=\bigcup_{H \in \Gamma} H$ is quasi-closed.Also for any $y \in X_{2}$ and $H \in \Gamma, f^{-1}(y) \cap H \neq \varnothing$. Because $f^{-1}(y)$ is $p$-compact, it follows that $f^{-1}(y) \cap F \neq \varnothing$. Hence $f(F)=X_{2}$. The lemma now follows by ap aplication of Zorn's lemma.

(5.5) Lemma. Let $f$ be a mapping from $\left(X_{1}, P_{1}, Q_{1}\right)$ onto $\left(X_{2}, P_{2}, Q_{2}\right)$ such that $f$ does not map any proper $P_{1}$-closed, $Q_{1}$-closed subset of $X_{1}$ onto $X_{2}$. Then $f$ does not map any proper semi-closed subset of $X_{1}$ onto $X_{2}$.

Proof. A semi-closed set is the intersection of finite unions of $P_{1}$-closed and $Q_{1}$-closed subset of $X_{1}$. Since no proper $P_{1}$-closed, $Q_{1}$-closed subset of $X_{1}$ is mapped onto $X_{2}$, it follows that no proper semi-closed subset of $X_{1}$ is mapped onto $X_{2}$. 
(5.6) LEMMA. Suppose that $f$ is a closed, continuous mapping from a space $\left(X_{1}, P_{1}, Q_{1}\right)$ onto a space $\left(X_{2}, P_{2}, Q_{2}\right)$. Suppose also that $f$ does not map any proper semi-closed subset of $X_{1}$ onto $X_{2}$. Then if $H$ is any $P_{1}$-open subset of $X_{1}$, $f(H) \subset\left[\left(f\left(H^{\prime}\right)\right)^{\prime}\right]^{Q_{2}}$ and if $K$ is any $Q_{1}$-open subset of $\left.X_{1}, f(K) \subset\left[f\left(K^{\prime}\right)\right)^{\prime}\right]^{P_{2}}$.

Proof. Considering the semi-open subset

$$
W=H \cap\left(f^{-1}\left[\left(f\left(H^{\prime}\right)\right)^{\prime}\right]^{Q_{2}}\right)^{\prime}
$$

of $X_{1}$ and proceeding in the similar way as that in lemma 6 of [7], we shall get

$$
f(H) \subset\left[\left(f\left(H^{\prime}\right)\right)^{\prime}\right]^{22} \text {. }
$$

Similarly, if $K$ is any $Q_{1}$-open subset of $X_{1}$, we have

$$
f(K) \subset\left[\left(f\left(K^{\prime}\right)\right)^{\prime}\right]^{P_{2}} .
$$

\section{6. p-Extremally disconnected spaces}

(6.1) We begin with two examples of $p$-extremally disconnected spaces.

EXAmple 1. Let $X$ be the real line $R$. Let $P$ be the usual topology and $Q$ be the discrete topology. Then $(X, P, Q)$ is $p$-extremally disconnected. It is p-Hausdorff and p-regular.

Example 2. Let $X$ be the real line $R$. Define $u$ by $u(x, y)=0$ if $y \geqq x, u(x, y)$ $=1$ if $y<x$ and $v$ by $v(x, y)=u(y, x)$. Let $P$ and $Q$ be the topologies generated by $u$ and $v$ respectively. Then $(X, P, Q)$ is $p$-extremally disconnected. It is not p-Hausdorff.

(6.2) LEMMA. In a p-extremally disconnected, p-Hausdorff space, if $U$ and $V$ are disjoint $P$-open and $Q$-open sets respectively, then $U^{Q} \cap V^{P}=\varnothing$.

The proof follows by lemma (3.1) and definition (2.2)

(6.3) Definition. A mapping $f:\left(X_{1}, P_{1}, Q_{1}\right) \rightarrow\left(X_{2}, P_{2}, Q_{2}\right)$ is said to be homeomorphism if $f$ is bijective and both $f$ and $f^{-1}$ are continuous.

(6.4) LEMMA. Let $f$ be a closed continuous mapping of a p-Hausdorff space $\left(X_{1}, P_{1}, Q_{1}\right)$ onto a p-extremally disconnected, p-Hausdorff space $\left(X_{2}, P_{2}, Q_{2}\right)$. If $f$ does not map any proper semi-closed subset of $X_{1}$ onto $X_{2}$, then $f$ is a homeomorphism.

The proof is similar to that of lemma 7 of [7].

\section{Proof of the main theorem}

The following proof runs on the same lines as that of Strauss [7].

Suppose that $f$ is a perfect mapping from a $p$-regular, $p$-Hausdorff space 
$\left(Y, P_{1}, Q_{1}\right)$ onto a $p$-regular, $p$-Hausdorff space $\left(X, P_{2}, Q_{2}\right)$; and that $g$ is a perfect mapping from a $p$-extremally disconnected, $p$-regular and $p$-Hausdorff space $\left(E, P_{3}, Q_{3}\right)$ into $\left(X, P_{2}, Q_{2}\right)$. We shall show that there is a perfect mapping $h$ from $E$ into $Y$ such that $f h=g$.

Consider the subset

$$
Z=\{(e, y): g(e)=f(y)\} \subset(E \times Y, P, Q)
$$

where $P=P_{3} \times P_{1}$ and $Q=Q_{3} \times Q_{1}$. We shall first show that the projection $\pi_{1}$ of $Z$ onto $E$ is perfect. It is clearly continuous for $\pi_{1}: E \times Y \rightarrow E$ is continuous. As

$$
\pi_{1}^{-1}(e)=\{e\} \times f^{-1}\{g(e)\},
$$

it is compact because by theorem (4.9), $\{e\} \times f^{-1}\{g(e)\}$ is p-compact; and $P$-compact and $Q$-compact by Tychonoff's theorem. We must show that $\pi_{1}$ is closed mapping. Let $F$ be a $P$-closed subset of $Z$ and let $e \in\left(\pi_{1}(F)\right)^{\prime}$. Then the $P$-compact set

$$
\{e\} \times f^{-1}\{g(e)\}
$$

and the $P$-closed set $F$ are disjoint subsets of $E \times Y$ and so by lemma (3.4), there exists a $P$-open set $H$ in $E \times Y$ such that

$$
\{e\} \times f^{-1}\{g(e)\} \subset H \text { and } F \cap H=\varnothing .
$$

Since $\{e\} \times f^{-1}\{g(e)\}$ is $P$-compact, for some integer $n$,

$$
\{e\} \times f^{-1}\{g(e)\} \subset \bigcup_{i=1}^{n}\left(D_{i} \times W_{i}\right) \subset H
$$

where $D_{i}$ is $P_{3}$-open subset of $E$ and $W_{i}$ is $P_{1}$-open subset of $Y$ for each $i=1$ to $n$. Now Strauss's [7] argument at this point may be repeated and we are led to prove that $\pi_{1}(F)$ is $P_{3}$-closed. Similarly, $\pi_{1}$ maps $Q$-closed subset of $Z$ into $Q_{3}$ closed subset of $E$. Hence $\pi_{1}$ is a closed mapping.

Similarly, the projection $\pi_{2}$ of $E \times Y$ onto $Y$ is perfect.

Now by lemma (5.4), there is a quasi closed subset $Z_{0}$ of $Z$ for which $\pi_{1}\left(Z_{0}\right)=E$ and no proper $P$-closed, $Q$-closed subset of $Z_{0}$ is mapped onto $E$. By lemma (5.5) no proper semi-closed subset of $Z_{0}$ is mapped onto $E$. By lemma (6.4), $\pi_{1} \mid Z_{0}$ is a homeomorphism and has an inverse say $\sigma$. Finally, if we put $h=\pi_{2} \sigma, h$ will be the required mapping.

Conversely, let $(X, P, Q)$ be a projective space in such a category. Let $G$ be a $P$-open subset of $X$. We shall show that $G^{Q}$ is $P$-open. Let $\left(Z, P_{1}, Q_{1}\right)$ be a $p$-regular, $p$-Hausdorff space and let $a, b$ be two distinct points of $Z$. Consider

$$
Y=\left(G^{\prime} \times\{a\}\right) \cup\left(G^{\mathcal{Q}} \times\{b\}\right) \subset X \times Z .
$$

Let $i$ be the inclusion map of $Y$ into $X \times Z$ and $\pi$ be the projection of $X \times Z$ onto $X$. Then it is easy to see that $\pi i$ is a perfect mapping from $Y$ onto $X$. Since $X$ 
is projective there exists a perfect map $\psi: X \rightarrow Y$ such that $\pi i \psi=I$, where $I$ is the identity map from $X$ onto $X . \pi i$ is one-one on $(G \times\{b\})$. Therefore $\psi(x)=(x, b)$. Since $\psi$ is continuous,

$$
\psi(x) \in(G \times\{b\})^{Q \times Q_{1}}
$$

for all $x \in G^{Q}$. But

$$
(G \times\{b\})^{Q \times Q_{1}} \subset G^{Q} \times\{b\} .
$$

Therefore $G^{Q} \subset \psi^{-1}\left(G^{Q} \times\{b\}\right)$. Also if $x \notin G^{Q}$ then $\psi(x)=(x, a)$. So,

$$
G^{Q}=\psi^{-1}\left(G^{Q} \times\{b\}\right) \text {. }
$$

But $\psi$ is continuous and $\left(G^{\prime} \times\{a\}\right)$ is $P$-closed in $Y$ and so $\left(G^{Q} \times\{b\}\right)$ is $P$-open in $Y$. Therefore $G^{Q}$ is $P$-open in $X$.

Similarly we can show that $P$-closure of any $Q$-open set is $Q$-open. Therefore $(X, P, Q)$ is $p$-extremally disconnected.

(7.1) Remark. The intersection of cat A and cat B is precisely the category of semi-compact, $p$-Hausdorff spaces and continuous maps. Since semi-compact spaces are $p$-compact, $P$-compact and $Q$-compact, it follows by theorem 10 of [2] that $P=Q$. Thus the intersection of cat $\mathrm{A}$ and cat $\mathrm{B}$ is precisely $\mathscr{G}$.

I express my deep sense of gratitude to Prof. V. Krishnamurthy for his encouragement and help in the preparation of this paper.

\section{References}

[1] M. C. Datta, 'Projective bitopological spaces', Journal of the Australian Math. Soc. 13 (1972), 327-334.

[2] P. Fletcher, H. B. Hoyle III and C. W. Patty, 'The comparison of topologies', Duke Math. Journ. 36 (1969), 325-332.

[3] A. M. Gleason, 'Projective topological spaces', Illi. Journ. Math. 2 (1958), 482-489.

[4] J. C. Kelly, 'Bitopological spaces', Proc. Lond. Math. Soc., 3, 13 (1963), 71-89.

[5] T. O. Moore, Elementary General Topology (Prentice-Hall, Englewood Cliffs, N. J., 1964).

[6] W. J. Pervin, 'Connectedness in bitopological spaces'. Ind. Math. 29 (1967), 369-372.

[7] D. P. Strauss, 'Extremally disconnected spaces', Proc. Amer. Math. Soc. 18 (1967) 305-309.

[8] W. J. Thron, Topological Structures (Holt, Rinehart and Winston, New York, 1966).

Department of Mathematics

Birla Institute of Technology and Science

Pilani (Rajasthan), India 